\title{
The zeta function of a quasi-ordinary singularity
}

\author{
Lee J. McEwan and András Némethi
}

\begin{abstract}
We prove that the zeta function of an irreducible hypersurface quasi-ordinary singularity $f$ equals the zeta function of a plane curve singularity $g$. If the local coordinates $\left(x_{1}, \ldots, x_{d+1}\right)$ of $f$ are 'nice', then $g=f\left(x_{1}, 0, \ldots, 0, x_{d+1}\right)$. Moreover, the Puiseux pairs of $g$ can also be recovered from (any set of) distinguished tuples of $f$.
\end{abstract}

\section{Introduction}

We consider the germ of an irreducible quasi-ordinary singularity $f:\left(\mathbb{C}^{d+1}, 0\right) \rightarrow(\mathbb{C}, 0)$. The quasi-ordinary assumption means that, in some local coordinates, the reduced discriminant of the projection $p r:(F, 0):=(\{f=0\}, 0) \rightarrow\left(\mathbb{C}^{d}, 0\right)$, induced by $\left(x, x_{d+1}\right) \mapsto x\left(x=\left(x_{1}, \ldots, x_{d}\right) \in \mathbb{C}^{d}\right)$, is included in $\left(\left\{x_{1} \cdots x_{d}=0\right\}, 0\right)$.

The main goal of this note is the computation of the zeta function of $f$. This is defined as follows.

For any germ $f:\left(\mathbb{C}^{d+1}, 0\right) \rightarrow(\mathbb{C}, 0)$, we fix a sufficiently small closed ball $B_{r}$ in $\mathbb{C}^{d+1}$ of radius $r$, then $F_{\epsilon}:=f^{-1}(\epsilon) \cap B_{r}(0<\epsilon \ll r)$ is called the Milnor fiber of $f$. In fact, by a theorem of Milnor [Mil68], $f^{-1}(\{|w|=\epsilon\}) \cap B_{r} \rightarrow\{|w|=\epsilon\}$ is a fibration with fiber $F_{\epsilon}$. Let $m_{q} \in$ Aut $H_{q}\left(F_{\epsilon}, \mathbb{R}\right)$ $(q \geqslant 0)$ be the algebraic monodromy operators of this fibration. Then the zeta function of $f$ is defined by the following rational function:

$$
\zeta(f)(t):=\prod_{q \geqslant 0} \operatorname{det}\left(I-t m_{q}\right)^{(-1)^{q}} .
$$

In general, $\zeta(f)$ is not computed by this expression. The most efficient way to determine $\zeta(f)$ is by A'Campo's formula ([A'C75], see also $\S 4$ ) via the embedded resolution of $f$. Hence, in the case of those families of singularities whose embedded resolution is well understood, one gets $\zeta(f)$. This is the case for plane curve singularities (see e.g. [EN85]) and isolated singularities with non-degenerate Newton boundary ([Var76], see also [MO70]). For non-isolated singularities, the methods of series of singularities, provide partial results (see e.g. [Sie90, Sch90, Ném93]) provided that the singular locus is one-dimensional. But, in general, for non-isolated singularities there is no nice, explicit formula of $\zeta(f)$.

We recall that in general the singular locus of quasi-ordinary singularities is large. Our main result for these singularities is the following.

Theorem A. Assume that $f:\left(\mathbb{C}^{d+1}, 0\right) \rightarrow(\mathbb{C}, 0)(d \geqslant 2)$ is an irreducible quasi-ordinary singularity. For convenience, we assume that the coordinates are ordered (cf. $\S 2.2)$. Then $\zeta(f)=\zeta\left(\left.f\right|_{x_{d}=0}\right)$. In fact, by induction

$$
\zeta(f)=\zeta\left(\left.f\right|_{x_{d}=\cdots=x_{2}=0}\right)
$$

Received 17 December 2001, accepted in final form 17 September 2002.

2000 Mathematics Subject Classification 14B05, 32Sxx.

Keywords: quasi-ordinary singularities, Milnor fiber, monodromy, zeta function, Newton polyhedron, toric resolution.

The second author is partially supported by NSF grant DMS-0088950.

This journal is (C) Foundation Compositio Mathematica 2004. 


\section{J. MCEWAN AND A. NÉmethi}

hence $\zeta(f)$ can be computed as the zeta function of the plane curve singularity $\left(x_{1}, x_{d+1}\right) \mapsto$ $f\left(x_{1}, 0, \ldots, 0, x_{d+1}\right)$.

Here some comments are in order. First, this type of result is rather surprising. In general, for germs $f$ with one-dimensional singular locus and generic hyperplane section $x_{d}$, an identity $\zeta(f)=\zeta\left(\left.f\right|_{x_{d}=0}\right)$ holds if the corresponding polar curve is empty. Our germ and hyperplane section are more complicated, but still the above theorem suggests the vanishing of some polar obstruction. This is a remarkable property of quasi-ordinary germs; the details will appear elsewhere.

We recall also that the quasi-ordinary singularities are generalizations of the plane curve singularities. For any ( $d$-dimensional) irreducible quasi-ordinary singularity $f$, one can define the generalization of the Puiseux pairs: they are called the normalized distinguished tuples $\left\{\lambda_{i}\right\}_{i=1}^{g}$ of $f$. (For details, see [Lip65, Lip83, Lip88].) Using Zariski's result on saturation of local rings, one can prove that these pairs determine the embedded topological type of $f$ (cf. [Zar68] and [Lip88], or [Oh93] for a different proof). They suffice as well to determine an embedded resolution (see [BMc00] and [GP00]). In addition, by [BMcN02] (cf. also with [LDT73] and [Tei74]), one has that, for any reduced hypersurface singularity, the zeta function depends only on the embedded topological type of the singularity. Therefore, it is natural to ask for explicit formulae for $\zeta(f)$ in terms of the distinguished tuples of $f$.

In the next section we reformulate Theorem A. This second version provides this explicit formula as well. It is remarkable that not all the data of the distinguished pairs are needed.

Theorem A is the consequence of Theorem B.

Theorem B. Let $\lambda_{1}$ be the first distinguished tuple of $f$. For any $\alpha \in \mathbb{Z}_{\geqslant 0}^{d}$ set $I(\alpha):=\left\{i \mid \alpha_{i}>0\right\}$. Assume that $0 \neq \beta \in \mathbb{Z}_{\geqslant 0}^{d}$ satisfies $\# I\left(\beta+\lambda_{1}\right) \geqslant 2$. Then

$$
\zeta\left(x^{\beta} f\right)(t)=1 \text {. }
$$

The precise definition of the distinguished tuples will be given in $\S 2$. As a general reference, see [Lip65, Lip83, Lip88]. The general properties of the Milnor fibration and smoothing invariants, in particular, of the zeta function, can be found in the books [Mil68], [EN85] and [AGV88].

The proof of Theorem B occupies all of $\S \S \S 3,4$ and 5 .

In $\S 3$ we recall some results from the thesis of González Pérez [GP00], namely the construction of a toric modification $\pi(\Sigma)$ associated with the first distinguished tuple $\lambda_{1}$ of $f$. Moreover, we add some new facts which will be important in our proof. For general facts about toric geometry, we recommend e.g. the books [Oda88] and [Ful93]. For the properties of the toric modification $\pi(\Sigma)$ see also [GT00].

In $\S 4$ we will consider a generalization of the classical A'Campo theorem [A'C75]. Using this we prove a 'splitting property' for the invariant $\zeta$, which is one of the key arguments in the proof of Theorem B; moreover, Theorem A is the consequence exactly of this property. At the end of this section we also recall the main result of [GLM97], which provides a formula for the zeta function in the presence of a partial embedded resolution. We will apply this result in the case of $\pi(\Sigma)$.

Section 5 contains the proof of the theorems collecting all the information from the previous sections.

Theorems $\mathrm{A}$ and $\mathrm{B}$ were conjectured in the article [BMcN02], where the authors proved the corresponding results for $d=2$ and the invariant $\chi\left(F_{\epsilon}\right)=\operatorname{deg}(\zeta(f))($ instead of $\zeta(f))$.

Finally, we note that in the literature the notation for the parametrization of a quasi-ordinary singularity is $\zeta$, and for the zeta function of a hypersurface singularity it is $\zeta(f)$. Even if these notations are almost identical, we do not modify them: the meaning of the corresponding notation will also be clear from the context. 


\section{THE ZETA FUNCTION OF A QUASI-ORDINARY SINGULARITY}

\section{Quasi-ordinary singularities and their topological type}

In this section we recall the definition of the distinguished tuples associated with an irreducible quasi-ordinary singularity $(F, 0) \subset\left(\mathbb{C}^{d+1}, 0\right)$, its connection with the embedded topological type of $(F, 0) \subset\left(\mathbb{C}^{d+1}, 0\right)$, and we reformulate our Theorem A.

As a general reference for quasi-ordinary singularities, see [Lip65, Lip83, Lip88].

2.1 We will use the notation $\bar{x}=\left(x, x_{d+1}\right)=\left(x_{1}, \ldots, x_{d}, x_{d+1}\right) \in \mathbb{C}^{d} \times \mathbb{C}=\mathbb{C}^{d+1}$ for coordinates in $\mathbb{C}^{d+1}$ (or for local coordinates in $\left(\mathbb{C}^{d+1}, 0\right)$ ).

By the very definition of an irreducible hypersurface quasi-ordinary singularity $f:\left(\mathbb{C}^{d+1}, 0\right) \rightarrow$ $(\mathbb{C}, 0)$, there exist local coordinates $\left(x, x_{d+1}\right)$ such that $f$ can be expressed as a (Weierstrass) pseudopolynomial in the variable $x_{d+1}$ :

$$
f\left(x, x_{d+1}\right)=x_{d+1}^{n}+g_{1}(x) x_{d+1}^{n-1}+\cdots+g_{n}(x),
$$

where $g_{i} \in \mathbb{C}\{x\}$ (= the ring of convergent power series in $x$ ), and the reduced discriminant of $p r:(\{f=0\}, 0) \rightarrow\left(\mathbb{C}^{d}, 0\right)$ induced by $\left(x, x_{d+1}\right) \mapsto x$ is contained in $\left(\left\{x_{1} \cdots x_{d}=0\right\}, 0\right)$. Moreover, by the Jung-Abhyankar Theorem [Abh55], there exists a parametrization of $(F, 0)=(\{f=0\}, 0)$ by a fractional power series $\zeta=H\left(x_{1}^{1 / m}, \ldots, x_{d}^{1 / m}\right)$, where $H\left(s_{1}, \ldots, s_{d}\right)$ is a power series and $m$ is a suitable natural number depending on $f$. (This means that there exists a finite map $\left(\mathbb{C}^{d}, 0\right) \rightarrow(F, 0)$ given by $x_{d+1}=H\left(s_{1}, \ldots, s_{d}\right), x_{i}=s_{i}^{m}$ for $i=1, \ldots, d$.)

The conjugates of $\zeta$ are obtained by multiplying any of $x_{i}^{1 / m}(i=1, \ldots, d)$ by $m$ th roots of unity; the number of different conjugates $\left\{\zeta_{i}\right\}_{i}$ of $\zeta$ is precisely the degree $n$ of the covering $p r$, and

$$
f\left(x, x_{d+1}\right)=\prod_{i=1}^{n}\left(x_{d+1}-\zeta_{i}\right) .
$$

There is an important finite subset $\left\{\lambda_{i}\right\}_{i=1}^{g}$ of the set of exponents $\lambda=\left(\lambda_{1}, \ldots, \lambda_{d}\right) \in \mathbb{Q}_{\geqslant 0}^{d}$ of the terms $x^{\lambda}:=x_{1}^{\lambda_{1}} \cdots x_{d}^{\lambda_{d}}$ appearing in $\zeta$ with non-zero coefficient, called the distinguished $d$-tuples associated with $\zeta$ (in some articles they are called characteristic exponents, see [GP00]). They play a role similar to Puiseux pairs for plane curve singularities.

By unique factorization of the discriminant one has

$$
\zeta_{i}-\zeta_{j}=x^{\lambda_{i j}} \cdot \epsilon_{i j}\left(x_{1}^{1 / m}, \ldots, x_{d}^{1 / m}\right) \text { with } \epsilon_{i j}(0) \neq 0,
$$

and $\lambda_{i j, k} \in(1 / m) \mathbb{Z}_{\geqslant 0}$. The set $\left\{\lambda_{i j}\right\}_{i j}=\left\{\left(\lambda_{i j, 1}, \ldots, \lambda_{i j, d}\right)\right\}_{i j}$ constitutes the set of distinguished tuples.

2.2 In the rest of this paper we use the following partial ordering. For any $\lambda, \mu \in \mathbb{Q}^{d}$, we say that $\lambda \leqslant \mu$ if $\lambda_{i} \leqslant \mu_{i}$ for all $i=1, \ldots, d$. If $\lambda \leqslant \mu$, but $\lambda \neq \mu$, then we write $\lambda<\mu$.

The distinguished tuples $\left\{\lambda_{i}\right\}_{i=1}^{g}$ are totally ordered:

$$
0<\lambda_{1}<\lambda_{2}<\cdots<\lambda_{g} .
$$

Let $\alpha_{\lambda}$ be the coefficient of the term $x^{\lambda}$ in $\zeta$ having exponent $\lambda$. Then the distinguished tuples $\left\{\lambda_{i}\right\}_{i=1}^{g}$ generate all the other exponents in the following sense:

$$
\text { If } \alpha_{\lambda} \neq 0 \quad \text { then } \lambda \in \mathbb{Z}^{d}+\sum_{\lambda_{i} \leqslant \lambda} \mathbb{Z}\left(\lambda_{i}\right) ; \quad \text { and } \quad \lambda_{j} \notin \mathbb{Z}^{d}+\sum_{\lambda_{i}<\lambda_{j}} \mathbb{Z}\left(\lambda_{i}\right) \quad \text { for } 1 \leqslant j \leqslant g .
$$

Sometimes it is convenient to order the coordinates $x_{1}, \ldots, x_{d}$ : after permuting $\left(x_{1}, \ldots, x_{d}\right)$ we can assume

$$
\left.\left(\lambda_{1,1}, \ldots, \lambda_{g, 1}\right)>\left(\lambda_{1,2}, \ldots, \lambda_{g, 2}\right)>\cdots>\left(\lambda_{1, d}, \ldots, \lambda_{g, d}\right) \quad \text { (lexicographically in } \mathbb{Q}^{g}\right) .
$$




\section{J. McEwAn AND A. NÉMEthi}

The parametrization $\zeta$ of $F$, in general, is not unique. Consider, for example, an arbitrary parametrization $\zeta$ as above. $\zeta$ can be written in a unique way as $\zeta^{(0)}+\zeta^{(>0)}$, so that $\zeta^{(0)} \in \mathbb{C}\{x\}$, and for any non-zero term $\alpha_{\lambda} x^{\lambda}$ of $\zeta^{(>0)}$ one has $\lambda \notin \mathbb{Z}^{d}$. We say that the local coordinates $\left(x, x_{d+1}\right)$ (of $\zeta$ ) are 'good' (cf. [GP00, p. 71], cf. also with [BMcN02, property (4)]) if

$$
\zeta^{(0)}=0 \text {. }
$$

Obviously, if the coordinates of $\zeta$ are not 'good', then the local change of variables $x_{d+1}^{\prime}=x_{d+1}-$ $\zeta^{(0)}, x_{i}^{\prime}=x_{i}$ for $i=1, \ldots, d$, transforms $\zeta$ into $\zeta^{\prime}=\zeta-\zeta^{(0)}$ with $\left(\zeta^{\prime}\right)^{(0)}=0$. This transformation preserves the distinguished tuples of the parametrizations.

In fact, different parametrizations could produce different sets of distinguished tuples. But if we consider only the 'normalized' parametrizations, then the corresponding tuples are independent of the choice of parametrization. A parametrization $\zeta$ is normalized if in addition to (1)-(3) we have the following:

$$
\text { If } \lambda_{1}=\left(\lambda_{1,1}, 0, \ldots, 0\right) \text { then } \lambda_{1,1}>1 \text {. }
$$

We recall the following facts about distinguished tuples.

2.3 By a result of Lipman [Lip65, Lip83, Lip88], by a suitable change of variables, any parametrization $\zeta$ can be transformed into a normalized parametrization $\zeta^{\prime}$. The normalized distinguished tuples of $\zeta^{\prime}$ (or of $f$ ) can be determined explicitly from the distinguished tuples of $\zeta$.

From [Lip88, Gau88] the embedded topological type of $f$ determines, and is determined by, the normalized distinguished tuples of $f$. In particular, via the above statement, any set of distinguished tuples (normalized or not) contains the complete information about the embedded topological type of $(F, 0) \subset\left(\mathbb{C}^{d+1}, 0\right)$.

We add to this list the following general fact about hypersurface singularities.

Let $f:\left(\mathbb{C}^{d+1}, 0\right) \rightarrow(\mathbb{C}, 0)$ be a reduced hypersurface singularity with Milnor fiber $F_{\epsilon}$ and geometric monodromy $m_{\text {geom }}: F_{\epsilon} \rightarrow F_{\epsilon}$ (defined up to an isotopy). Then the homotopy type of the pair $\left(F_{\epsilon}, m_{\text {geom }}\right)$ can be recovered from the embedded topological type of $f$. (For isolated singularities it was proved in [LDT73], cf. also [Yau89] and [Tei74]; in the general case by [BMcN02].)

2.4 The above results show that there should exist an explicit formula of $\zeta(f)$ of any irreducible quasi-ordinary singularity $f$ in terms of the distinguished tuples $\left\{\lambda_{i}\right\}_{i=1}^{g}$ of any parametrization $\zeta$ (normalized or not) of $(F, 0)$. Our main theorem provides such a formula.

Theorem A (reformulated). Assume that $f:\left(\mathbb{C}^{d+1}, 0\right) \rightarrow(\mathbb{C}, 0)(d \geqslant 2)$ is an irreducible quasiordinary singularity represented in a local coordinate system $\left(x, x_{d+1}\right)$ as in $\S 2.1$. For convenience, we assume that the coordinates are ordered, i.e. (3) is satisfied. Then $\zeta(f)=\zeta\left(\left.f\right|_{x_{d}=0}\right)$, hence (by induction):

$$
\zeta(f)=\zeta\left(\left.f\right|_{x_{d}=\cdots=x_{2}=0}\right) .
$$

Moreover, if the first distinguished tuple $\lambda_{1}=\left(\lambda_{1,1}, \ldots, \lambda_{1, d}\right)$ satisfies $\lambda_{1,2} \neq 0$, then

$$
\left.f\right|_{x_{d}=\cdots=x_{2}=0}=\left(x_{d+1}-\zeta^{(0)}\left(x_{1}, 0, \ldots, 0\right)\right)^{n},
$$

hence $\zeta(f)(t)=1-t^{n}$.

If $\lambda_{1,2}=0$ (hence $\lambda_{1}=\left(\lambda_{1,1}, 0, \ldots, 0\right)$ ), then let $i_{0} \geqslant 1$ be that index which satisfies $\lambda_{i_{0}, 2}=0$ but $\lambda_{i_{0}+1,2} \neq 0$ (if $\lambda_{i, 2}=0$ for all $i=1, \ldots, g$, then $i_{0}=g$ ). Then

$$
\left.f\right|_{x_{d}=\cdots=x_{2}=0}=\left(\tilde{f}\left(x_{1}, x_{d+1}\right)\right)^{n / \operatorname{deg} \tilde{f}},
$$

where $\tilde{f}$ is an irreducible plane curve singularity, which has the equisingularity type of the singular germ defined by the Puiseux parametrization $x_{d+1}=\sum_{i=1}^{i_{0}} x_{1}^{\lambda_{i, 1}}$, and $\operatorname{deg} \tilde{f}$ is the $x_{d+1}$-degree of $\tilde{f}$. 
Remark. It is well known that, for any germ $\tilde{f}$ and integer $s \geqslant 1$, one has

$$
\zeta\left(\tilde{f}^{s}\right)(t)=\zeta(\tilde{f})\left(t^{s}\right)
$$

On the other hand, for any irreducible plane curve singularity $\tilde{f}, \zeta(\tilde{f})$ has an explicit expression in terms of the Puiseux pairs, see e.g. [EN85]. Moreover, $\operatorname{deg} \tilde{f}$ can also be determined from the exponents $\left\{\lambda_{i, 1}\right\}_{i=1}^{i_{0}}$ : it is the number of conjugates of $\sum_{1 \leqslant i \leqslant i_{0}} x_{1}^{\lambda_{i, 1}}$.

\section{The partial toric resolution: results of González Pérez}

In this section we will assume that $f$ is a quasi-ordinary singularity as in $\S 2.1$, satisfying property (4). (Note that properties (1) and (2) are automatically satisfied.) We will consider a toric modification $\pi(\Sigma): Z(\Sigma) \rightarrow \mathbb{C}^{d+1}$ whose restriction above a small ball $B_{r}$ provides a partial resolution of $(F, 0):=\left(f^{-1}(0), 0\right) \subset\left(\mathbb{C}^{d+1}, 0\right)$. In order to be uniform in the next discussions, we will use $\mathbb{C}^{d+1}$ instead of $B_{r}$, but this will not affect the proof of our theorems (since in the formula given in Theorem 4.7 only $\pi(\Sigma)^{-1}(0)$ is used; see also Remark 4.6).

Some results of this section are proved in the thesis of González Pérez [GP00]. We will indicate the corresponding page numbers at the corresponding places.

\subsection{The function $f_{1}$}

Let $f$ be as above. Properties (2) and (4) guarantee that, if the coefficient $\alpha_{\lambda}$ of $x^{\lambda}$ in the parametrization $\zeta$ is non-zero, then $\lambda_{1} \leqslant \lambda$, i.e.

$$
\zeta=\alpha_{\lambda_{1}} x^{\lambda_{1}}+\sum_{\lambda_{1}<\lambda} \alpha_{\lambda} x^{\lambda} \quad\left(\alpha_{1}:=\alpha_{\lambda_{1}} \neq 0\right)
$$

Define $\zeta^{\prime}:=\alpha_{1} x^{\lambda_{1}}$. Obviously, $\zeta^{\prime}$ determines a quasi-ordinary singularity, which has only one distinguished tuple (namely $\lambda_{1}$ ), and degree $n_{1}=$ the number of conjugates of $x^{\lambda_{1}}$. Its monic minimal polynomial is

$$
f_{1}=\prod_{i=1}^{n_{1}}\left(x_{d+1}-\omega_{i} \alpha_{1} x^{\lambda_{1}}\right)=x_{d+1}^{n_{1}}-\alpha_{1}^{n_{1}} x^{n_{1} \lambda_{1}},
$$

where $\left\{\omega_{i}\right\}_{i}$ are the $n_{1}$ roots of unity. If $L$ denotes the fraction field of $\mathbb{C}\{x\}$, then $n=[L(\zeta): L]=$ $\left[L\left(x^{\lambda_{1}}, \ldots, x^{\lambda_{g}}\right): L\right]$, and $n_{1}=\left[L\left(x^{\lambda_{1}}\right): L\right]$. Therefore, $e_{1}:=n / n_{1} \in \mathbb{Z}_{>0}$.

\subsection{The Newton polyhedron of $f$}

Given $f=\sum_{v} c_{v} \bar{x}^{v} \in \mathbb{C}\{\bar{x}\}$, its Newton polyhedron $\mathcal{N}(f)$ is the convex hull of the set $\sum_{c_{v} \neq 0} v+$ $\mathbb{R}_{\geqslant 0}^{d+1}$. Any vector $u \in\left(\mathbb{R}^{d+1}\right)_{\geqslant 0}^{*}$ defines the face $\mathcal{F}_{u}$ of $\mathcal{N}(f)$ by

$$
\mathcal{F}_{u}:=\left\{v \in \mathcal{N}(f):\langle u, v\rangle=\inf _{v^{\prime} \in \mathcal{N}(f)}\left\langle u, v^{\prime}\right\rangle\right\} .
$$

If $f$ is a quasi-ordinary singularity as above, then $\mathcal{N}(f)$ has only two vertices, namely $(0, \ldots, 0, n)$ and $\left(n \lambda_{1,1}, \ldots, n \lambda_{1, d}, 0\right)$. Let $\mathcal{F}_{\text {co }}$ be the segment determined by these two vertices. Notice that $\mathcal{F}_{\text {co }}$ and its vertices are the only compact faces of $\mathcal{N}(f)$. By $(6)$ one has

$$
\left.f\right|_{\mathcal{F}_{\text {co }}}=\left(f_{1}\right)^{e_{1}} \text {. }
$$

Above $\left.f\right|_{\mathcal{F}}$ denotes the (symbolic) restriction of $f$ to the face $\mathcal{F}$, namely $\left.f\right|_{\mathcal{F}}=\sum_{v \in \mathcal{F}} c_{v} \bar{x}^{v}$. 


\section{J. McEwAn AND A. NÉMEthi}

\subsection{The fan $\Sigma(\mathcal{N}(f))$ in $\left(\mathbb{R}^{d+1}\right)_{\geqslant 0}^{*}$}

We say that two vectors in $\left(\mathbb{R}^{d+1}\right)_{\geqslant 0}^{*}$ are related if they define the same face in $\mathcal{N}(f)$. The fan $\Sigma(\mathcal{N}(f))$ is defined in such a way that the classes of the above relation are the relative interiors of the cones of $\Sigma(\mathcal{N}(f))$. In general, $\Sigma(\mathcal{N}(f))$ is not regular. We fix a regular fan $\Sigma$, supported by $\left(\mathbb{R}^{d+1}\right)_{\geqslant 0}^{*}$, which subdivides $\Sigma(\mathcal{N}(f))$. This, in particular, means that the intersection of any cone $\sigma \in \Sigma$ with the linear subspace $l$ given by the equation

$$
l(v):=\sum_{j=1}^{d} \lambda_{1, j} v_{j}-v_{d+1}=0
$$

is a cone of $\Sigma$. For any $\sigma \in \Sigma$, we denote by $\mathcal{F}_{\sigma}$ the face $\mathcal{F}_{u}$ of any vector $u$ from the relative interior of $\sigma$.

3.4 The regular fan $\Sigma$ supported by $\left(\mathbb{R}^{d+1}\right)_{\geqslant 0}^{*}$ defines a modification $\pi(\Sigma): Z(\Sigma) \rightarrow \mathbb{C}^{d+1}$. The variety $Z(\Sigma)$ is smooth, and it is covered by affine spaces $\{Z(\sigma)\}_{\operatorname{dim} \sigma=d+1}$ (i.e. each $Z(\sigma) \approx \mathbb{C}^{d+1}$ ). The inclusion $\sigma \subset\left(\mathbb{R}^{d+1}\right)_{\geqslant 0}^{*}$ provides a morphism $\pi(\sigma): Z(\sigma) \rightarrow \mathbb{C}^{d+1}$. If $\sigma=\left\langle a^{1}, \ldots, a^{d+1}\right\rangle$ and $a^{i}$ has coordinates $\left(a_{1}^{i}, \ldots, a_{d+1}^{i}\right)$, then $\pi(\sigma)$ has the form:

$$
x_{1}=u_{1}^{a_{1}^{1}} \cdots u_{d+1}^{a_{1}^{d+1}}, \ldots, x_{d+1}=u_{1}^{a_{d+1}^{1}} \cdots u_{d+1}^{a_{d+1}^{d+1}} .
$$

The total transform of $f=\sum_{v} c_{v} \bar{x}^{v}$ by $\pi(\sigma)$ is

$$
f \circ \pi(\sigma)=\sum_{v} c_{v} u_{1}^{\left\langle a^{1}, v\right\rangle} \cdots u_{d+1}^{\left\langle a^{d+1}, v\right\rangle}=u_{1}^{m\left(a^{1}\right)} \cdots u_{d+1}^{m\left(a^{d+1}\right)} \cdot \tilde{f}_{\sigma},
$$

where $m(a):=\inf _{v \in \mathcal{N}(f)}\langle a, v\rangle$ and $\tilde{f}_{\sigma}$ defines the equation of the strict transform of $f$ in the chart $Z(\sigma) \subset Z(\Sigma)$.

If $l(a) \geqslant 0$ (cf. Equation $(9)$ ), then $(0, \ldots, 0, n) \in \mathcal{F}_{a}$, hence

$$
m(a)=n a_{d+1} \text {. }
$$

\subsection{The divisors $D(a)$ in $Z(\Sigma)$}

To any primitive vector $a$ of $\Sigma^{(1)}(:=1$-skeleton of $\Sigma)$, we associate a divisor $D(a)$ in $Z(\Sigma)$. In any chart $Z(\sigma)$, associated with a cone $\sigma=\left\langle a^{1}, \ldots, a^{d+1}\right\rangle, D\left(a^{i}\right)$ is given by $\left\{u_{i}=0\right\}$. If $b \notin$ $\left\{a^{1}, \ldots, a^{d+1}\right\}$, then $D(b) \cap Z(\sigma)=\emptyset$. By (10) one gets that $\pi(\Sigma)\left(D\left(a^{i}\right)\right)$ is the coordinate subspace defined by $x_{j}=0$ for all $j$ with $a_{j}^{i} \neq 0$. If $e^{1}, \ldots, e^{d+1}$ denote the canonical basis on $\mathbb{Z}^{d+1}$, then $\pi(\Sigma)\left(D\left(e^{i}\right)\right)=\left\{x_{i}=0\right\}$, but for any $a \in \Sigma^{(1)} \backslash\left\{e^{1}, \ldots, e^{d+1}\right\}$ one has $\operatorname{dim} \pi(\Sigma)(D(a)) \leqslant d-1$. The critical locus (exceptional divisor) of $\pi(\Sigma)$ is exactly the union of these divisors:

$$
\bigcup_{a \in \Sigma^{(1)} \backslash\left\{e^{i}\right\}_{i}} D(a) .
$$

Moreover, $D(a)$ is compact (i.e. $D(a) \subset \pi(\Sigma)^{-1}(0)$ ) if and only if $a$ is an interior point of $\left(\mathbb{R}^{d+1}\right)_{\geqslant 0}^{*}$. More generally, one has the following fact.

Lemma 3.1. Let $A=\left\{a^{1}, \ldots, a^{s}\right\}$ be a non-empty subset of $\Sigma^{(1)}$. Denote $D_{A}:=\bigcap_{a \in A} D(a)$. Then:

a) if $D_{A} \neq \emptyset$, then $\left\{a^{1}, \ldots, a^{s}\right\}$ forms a cone $\sigma_{A}$ in $\Sigma$. Moreover, $L_{A}:=\pi(\Sigma)\left(D_{A}\right)$ is a linear coordinate subspace of $\mathbb{C}^{d+1}$;

b) assume that $D_{A} \neq \emptyset$, then

$$
D_{A} \text { compact } \Leftrightarrow \sigma_{A} \cap\left(\mathbb{R}^{d+1}\right)_{>0}^{*} \neq \emptyset \Leftrightarrow L_{A}=0 .
$$

The proof follows from standard facts of toric geometry and the previous discussion. 
3.6 The divisor $D:=\bigcup_{a \in \Sigma^{(1)}} D(a)$ in the smooth variety $Z(\Sigma)$ is a normal crossing divisor with smooth irreducible components. González Pérez proves [GP00, Theorem 3.6] that $\pi(\Sigma): Z(\Sigma) \rightarrow$ $\mathbb{C}^{d+1}$ provides an embedded resolution of $f_{1}$. If $\tilde{F}_{1}$ denotes the strict transform of $F_{1}=\left\{f_{1}=0\right\}$, then $\tilde{F}_{1}$ is smooth and $D \cup \tilde{F}_{1}$ is a normal crossing divisor. Moreover, the restriction $\left.\pi(\Sigma)\right|_{\tilde{F}_{1}}$ : $\tilde{F}_{1} \rightarrow F_{1}$ is an isomorphism above $F_{1} \backslash \operatorname{Sing} F_{1}$ (cf. [GP00, Lemma 3.6]). But, for a general $\Sigma$ (e.g. if $\Sigma^{(1)} \cap\left\{v^{d+1}=0\right\}$ is 'too rich'), it is not true that $\pi(\Sigma): Z(\Sigma) \rightarrow \mathbb{C}^{d+1}$ is an isomorphism above $\mathbb{C}^{d+1} \backslash \operatorname{Sing} F_{1}$. On the other hand, in the proof of Theorem B, the property that $\pi(\Sigma)$ is an isomorphism (at least) above $\mathbb{C}^{d+1} \backslash F_{1}$ (respectively above $\mathbb{C}^{d+1} \backslash F$ ) is crucial (cf. Theorem 4.7). (We notice that a similar property is used in [Var76] as well.)

Proposition 3.2. There exists a regular fan $\Sigma$ which subdivides $\Sigma(\mathcal{N}(f))$, supported by $\left(\mathbb{R}^{d+1}\right)_{\geqslant 0}^{*}$, so that $\pi(\Sigma)$ is an isomorphism above $\mathbb{C}^{d+1} \backslash\left(F_{1} \cap F\right)$. This means that the image

$$
\Delta_{\Sigma}:=\pi(\Sigma)\left(\bigcup_{a \in \Sigma^{(1)} \backslash\left\{e^{i}\right\}_{i}} D(a)\right)
$$

of the exceptional divisor of $\pi(\Sigma)$ is included in $F_{1} \cap F$.

Proof. A) First we assume that $\lambda_{1, i} \neq 0$ for all $i$; and we construct a regular fan $\Sigma$ with $\Delta_{\Sigma} \subset F_{1}$. Define $H:=\left\{v \in\left(\mathbb{R}^{d+1}\right)_{\geqslant 0}^{*}: v_{d+1}=0\right\}$. By assumption about $\lambda_{1}$ one has

$$
l \cap H \cap\left(\mathbb{R}^{d+1}\right)_{\geqslant 0}^{*}=0 .
$$

In [Ful93, pp. 47-48], Fulton gives the following algorithm to find a regular refinement $\Sigma$. This algorithm provides our wanted fan as well.

Given a fan $\Sigma_{0}$ and any lattice point $v$, one can subdivide $\Sigma_{0}$ to a fan $\Sigma_{1}$ as follows: each cone that contains $v$ is replaced by the joins of its faces with the ray through $v$; each cone not containing $v$ is left unchanged.

Now, the construction of the regular refinement is done by adding successively vectors $v$ as above. In this procedure there are two steps.

Step 1 . On can subdivide any fan by successively adding vectors in larger and larger cones, until it becomes simplicial.

Step 2. Any additional new vector $v$ has the following form: for some simplicial $k$-cone $\sigma$ generated by the primitive vectors $v_{1}, \ldots, v_{k}$, the new $v$ is a lattice point of the form $v=\sum_{i=1}^{k} t_{i} v_{i}\left(0 \leqslant t_{i}<1\right)$.

We have to show that, at any time in this procedure, one can make the choice of the vector $v$ so that $v \notin H$. In this case, for any $a \in \Sigma^{(1)} \backslash\left\{e^{i}\right\}_{i}$, we will have $a_{d+1} \neq 0$, hence $\pi(\Sigma)(D(a)) \subset F_{1}$ (cf. $\S 3.5)$.

Since $H \cap \Sigma(\mathcal{N}(f))$ is simplicial (cf. (13)), we can assume that all the vectors $v$ introduced in Step 1 have $v_{d+1}>0$. For Step 2, notice that any vector in $H \cap\left\{v_{1}, \ldots, v_{k}\right\}$ is automatically of type $\left\{e^{i}\right\}_{i \leqslant d}$, and the new (lattice) vector $v$ cannot be of the form $\sum_{i \leqslant d} t_{i} e^{i}\left(0 \leqslant t_{i}<1\right)$. Hence $v_{d+1}>0$.

B) Assume that $\lambda_{1, j_{0}} \neq 0$, but $\lambda_{1, j_{0}+1}=0$ for some $j_{0}<d$. First construct a refinement $\Sigma_{j_{0}}$ of $\Sigma(\mathcal{N}(f)) \cap\left(\mathbb{R}^{j_{0}+1}\right)_{\geqslant 0}^{*}$ in $\left(\mathbb{R}^{j_{0}+1}\right)_{\geqslant 0}^{*}$ (with coordinates $\left.v_{1}, \ldots, v_{j_{0}}, v_{d+1}\right)$, then define $\Sigma$ as the fan generated by $\Sigma_{j_{0}}$ and the vectors $\left\{e^{i}\right\}_{j_{0}<i \leqslant d}$. Then again $\Delta_{\Sigma} \subset F_{1}$.

C) Consider the linear coordinate subspace $L_{a}:=\pi(\Sigma)(D(a))$. Then it is easy to verify (using (6)) that if $L_{a} \subset F_{1}$ then $L_{a} \subset F$ as well. 


\section{J. MCEWAN AND A. NÉmethi}

\subsection{The strict transforms $\tilde{\boldsymbol{F}}_{1}$ and $\tilde{\boldsymbol{F}}$}

Let $g$ denote one of the functions $f$ or $f_{1}$, and set $\tilde{S}$ for the strict transform of $\{g=0\}$ via $\pi(\Sigma)$. In this section we will analyze the intersections $D \cap \tilde{S}$.

By [GP00, (3.2)], a divisor $D(a)\left(a \in \Sigma^{(1)}\right)$ has a non-empty intersection with $\tilde{S}$ if and only if $a \in l$. In fact, the intersection $D \cap \tilde{S}$ can be covered by charts $\{Z(\sigma)\}_{\sigma}$, where we consider only those $(d+1)$-dimensional cones $\sigma=\left\langle a^{1}, \ldots, a^{d+1}\right\rangle$ of $\Sigma$ which satisfy $a^{i} \in l$ for all $i=1, \ldots, d$, and $l\left(a^{d+1}\right)>0$ (cf. [GP00, page 110]). Therefore, all the properties of the intersections $D \cap \tilde{F}_{1}$ and $D \cap \tilde{F}$ can be verified in these charts.

First we analyze the intersection $D \cap \tilde{F}_{1}$.

Fix a chart $Z(\sigma)$ as above. Using $\mathcal{N}\left(f_{1}\right)=\left(1 / e_{1}\right) \mathcal{N}(f)$ and (12), the analog of (11) written for $f_{1}$ (and in $\left.Z(\sigma)\right)$ reads as

$$
f_{1} \circ \pi(\sigma)=\left(u_{1}^{a_{d+1}^{1}} \cdots u_{d+1}^{a_{d+1}^{d+1}}\right)^{n_{1}}\left(1-\alpha_{1}^{n_{1}} u_{d+1}^{n_{1} l\left(a^{d+1}\right)}\right),
$$

where $n_{1} l\left(a^{d+1}\right)=1$ (cf. [GP00, p. 111]). Therefore:

$$
\left(f_{1}\right)_{\sigma}^{\sim}=1-\alpha_{1}^{n_{1}} u_{d+1} .
$$

This means that $\tilde{F}_{1} \cap Z(\sigma)$ has equation $w:=1-\alpha_{1}^{n_{1}} u_{d+1}=0$. Notice that this equation is independent of the choice of $\sigma$. (Indeed, $u_{d+1}$, as a vector of $\sigma^{\sim}$, is a primitive vector generating $l^{\perp}$, cf. [Ful93, ch. 1].) This has the following important consequence. We say that the 'natural stratification' of $D \cap \tilde{F}_{1}$ is given by (non-empty) strata of type:

$$
\left(\bigcap_{a \in A} D(a) \backslash \bigcup_{a \notin A} D(a)\right) \cap \tilde{F}_{1} \quad\left(A \subset \Sigma^{(1)}\right) .
$$

Then the above discussion guarantees that the natural stratification of $D \cap \tilde{F}_{1}$ is equivalent to the stratification of the toroidal embedding whose combinatorics corresponds to the cones of $\Sigma \cap l$ (cf. [GP00, 3.2.3 and 3.3.2]). In particular,

$$
\text { for any stratum } \Xi \text { of } D \cap \tilde{F}_{1} \text { with } \operatorname{dim} \Xi>0 \text {, one has } \chi(\Xi)=0 \text {. }
$$

This can be explained in the following way as well. If a stratum $\Xi$ of $D \cap \tilde{F}_{1}$ has a non-empty intersection with the chart $Z(\sigma)$, then it is completely contained in $Z(\sigma)$, and in this chart is given by the intersection $\Xi_{D} \cap\{w=0\}$, where $\Xi_{D}$ is a toric stratum (orbit) of $D$ contained in $Z(\sigma)$. Hence $\Xi$ itself is a torus.

By similar argument as in Lemma 3.1, and using the above discussion, one obtains the following lemma.

Lemma 3.3. For any $A=\left\{a^{1}, \ldots, a^{s}\right\} \subset \Sigma^{(1)}$, one has the following.

a) If $D_{A} \cap \tilde{F}_{1} \neq \emptyset$, then $\left\{a^{1}, \ldots, a^{s}\right\}$ is a cone $\sigma_{A}$ in $\Sigma \cap l$.

b) Assume that $D_{A} \cap \tilde{F}_{1}$ is not empty. Then

$$
D_{A} \cap \tilde{F}_{1} \text { is compact } \Leftrightarrow \sigma_{A} \cap l \cap\left(\mathbb{R}^{d+1}\right)_{>0}^{*} \neq \emptyset .
$$

In particular, we obtain via Lemma 3.1, that

c) $D_{A} \cap \tilde{F}_{1}$ compact $\Leftrightarrow D_{A}$ compact.

The point is that these facts are true for the strict transform $\tilde{F}$ of $\{f=0\}$ as well.

Lemma 3.4. The statement of Lemma 3.3 is also true for $\tilde{F}$ instead of $\tilde{F}_{1}$.

Proof. a) is clear from the above discussion. By (8), $f$ is a perturbation of $f_{1}^{e_{1}}$. Using the local equations of the corresponding strict transforms, one obtains that, if $D_{A} \cap \tilde{F}_{1}$ is not compact, then $D_{A} \cap \tilde{F}$ cannot be compact either. Hence the lemma follows from Lemmas 3.1 and 3.3. 
The final goal of the above discussions is the next result.

Proposition 3.5. Assume that for a non-empty $A \subset \Sigma^{(1)}$ the intersection $D_{A}$ is non-empty and compact. Then

$$
D_{A} \cap \tilde{F}_{1}=D_{A} \cap \tilde{F} .
$$

This (via Lemmas 3.3 and 3.4) gives that:

$$
\pi(\Sigma)^{-1}(0) \cap \tilde{F}_{1}=\pi(\Sigma)^{-1}(0) \cap \tilde{F} .
$$

Proof. By Lemmas 3.1, 3.3 and 3.4 we can assume that $A$ defines a cone $\sigma_{A}$ in $l$ in such a way that $\sigma_{A} \cap l \cap\left(\mathbb{R}^{d+1}\right)_{>0}^{*} \neq \emptyset$. This implies that the face $\mathcal{F}_{\sigma_{A}} \subset \mathcal{N}(f)$ associated with $\sigma_{A}$ is compact, and in fact it is exactly $\mathcal{F}_{\text {co }}$. Then Proposition 3.5 follows from (8) and from the next technical lemma.

Lemma 3.6 [GP00, p. 105]. Set $f=\sum_{v} c_{v} \bar{x}^{v} \in \mathbb{C}\{\bar{x}\}$, and fix a compact face $\mathcal{F}_{\sigma}$ associated with the cone $\sigma=\left\langle a^{1}, \ldots, a^{s}\right\rangle \in \Sigma$. Then in $Z(\sigma)$, the intersection of the strict transform of $\{f=0\}$ with $\bigcap_{1 \leqslant i \leqslant s} D\left(a^{i}\right)$ has equation $\left(\left.f\right|_{\mathcal{F}_{\sigma}}\right)^{\sim}=0$.

\subsection{The strict and total transforms of $f$ along $\pi(\Sigma)^{-1}(0) \cap \tilde{F}$}

The divisor $D \cap \tilde{F}$ (also) has a 'natural stratification' given by strata of type

$$
\left(\bigcap_{a \in A} D(a) \backslash \bigcup_{a \notin A} D(a)\right) \cap \tilde{F} \quad\left(A \subset \Sigma^{(1)}\right) .
$$

We are interested only in those strata which are situated in $\pi(\Sigma)^{-1}(0)$. They are situated in compact intersections of type $D_{A}$ by Lemmas 3.3 and 3.4. Consider such a (connected) strata $\Xi$ with $\operatorname{dim} \Xi>0$. We claim that both the strict and the total transforms of $f$ along $\Xi$ determine an equisingular family of singularities. This follows from [GP00], which provides a simultaneous resolution of the strict transform of $f$ along $\Xi$. This shows in particular that the zeta function of the strict transform along $\Xi$ is constant.

3.9 Now we concentrate our discussion on the zero-dimensional strata $\{\Xi\} \operatorname{dim} \Xi=0$ of $D \cap \tilde{F}$. Obviously, all of these points are in $\pi(\Sigma)^{-1}(0) \cap \tilde{F}$.

For this, we fix an arbitrary $d$-cone $\sigma^{\prime}=\left\langle a^{1}, \ldots, a^{d}\right\rangle$ of $l \cap \Sigma$, and we consider the unique $(d+1)$ cone $\sigma=\left\langle a^{1}, \ldots, a^{d}, a^{d+1}\right\rangle$ of $\Sigma$ with a primitive vector $a^{d+1}$ satisfying $l\left(a^{d+1}\right)>0$. Then, in the chart $Z(\sigma)$ one can find a zero-dimensional stratum $O_{\sigma^{\prime}}:=\tilde{F} \cap D\left(a^{1}\right) \cap \cdots \cap D\left(a^{d}\right)$ of $D \cap \tilde{F}$.

In fact, there is a one-to-one correspondence between the zero-dimensional strata of $D \cap \tilde{F}$ and the points $O_{\sigma^{\prime}}$ where $\sigma^{\prime}$ runs over the $d$-cones of $\Sigma \cap l$.

By $\oint 3.7, O_{\sigma^{\prime}}$ is given by the equations $u_{1}=\cdots=u_{d}=0$ and $w:=1-\alpha_{1}^{n_{1}} u_{d+1}=0$. González Pérez in his thesis [GP00] shows that the strict transform $\tilde{f}_{\sigma}$ of $f$ in the local coordinates $\left(u_{1}, \ldots, u_{d}, w\right)$ is a quasi-ordinary singularity, but this coordinate system is not 'good' (cf. (4)). However, we can construct a local coordinate system $\left(u_{1}, \ldots, u_{d}, w_{1}\right)$ (i.e. only $w$ is modified, cf. $(4))$ in such a way that the projection $\left(u, w_{1}\right) \mapsto u$ induces a quasi-ordinary projection of $\left(\tilde{f}_{\sigma}, O_{\sigma^{\prime}}\right)$, which satisfies (1), (2) and (4). (In fact, by [GP00], $w_{1}=0$ is the strict transform of the hypersurface given by the vanishing of the first approximate root of $f$. $)$ The germ $\left(\tilde{f}_{\sigma}, O_{\sigma^{\prime}}\right)$ has smaller complexity than the original germ $f$ : it has only $g-1$ distinguished tuples. (They can be computed from the original tuples of $f$, but this formula is not essential here.)

\subsection{The total transform of $x^{\beta} f$}

Fix $\beta \in \mathbb{Z}_{\geqslant 0}^{d}$, and consider the germ $x^{\beta} f$. Notice that $\pi(\Sigma)^{-1}\left(\left\{x_{i}=0\right\}\right)=D\left(e^{i}\right)($ for $i=1, \ldots, d)$, hence $D \cup \tilde{F}=D \cup\left\{\right.$ the total transform of $\left.x^{\beta} f\right\}$, and both of them have the same "natural stratification' (i.e. by introducing the factor $x^{\beta}$, we do not create new strata). 


\section{J. MCEwan AND A. NÉmethi}

In the proof of Theorem B, we will use an inductive step, which replaces the germ $x^{\beta} f$ by its total transform at the points $\left\{O_{\sigma^{\prime}}\right\}_{\sigma^{\prime}}$. Obviously, the total transform of $x^{\beta} f$ at the point $O_{\sigma^{\prime}}$ has similar form, namely $u^{\gamma} \tilde{f}_{\sigma}$ for some $\gamma$ (cf. (11)).

In the next lemma we will use the following notation: For any $\alpha=\left(\alpha_{1}, \ldots, \alpha_{d}\right) \in \mathbb{Z}_{\geqslant 0}^{d}$, we define $I(\alpha):=\left\{i \mid \alpha_{i}>0\right\}$.

Proposition 3.7. Let $\sigma^{\prime}$ be a cone as in $\S$ 3.9. Let $u^{\gamma} \tilde{f}_{\sigma}$ be the total transform of $x^{\beta} f$ at the point $O_{\sigma^{\prime}}$. Then if $\# I(\beta) \geqslant 1$ and $\# I\left(\beta+\lambda_{1}\right) \geqslant 2$, then $\# I(\gamma) \geqslant 2$. (In particular, $u^{\gamma} \tilde{f}_{\sigma}$ satisfies the inductive property $\# I(\gamma) \geqslant 1$ and $\# I\left(\gamma+\lambda_{1}\left(\tilde{f}_{\sigma}, O_{\sigma^{\prime}}\right)\right) \geqslant 2$.)

Proof. For simplicity we write $\left\langle a^{i}, \beta\right\rangle=\sum_{j=1}^{d} a_{j}^{i} \beta_{j}$. Moreover, assume that the coordinates are ordered, i.e. (3) is satisfied as well.

Then by (10)-(12) one gets that

$$
\gamma_{i}=n a_{d+1}^{i}+\left\langle a^{i}, \beta\right\rangle, i=1, \ldots, d .
$$

If $a_{d+1}^{i}=0$ for all $i=1, \ldots, d$, then $a^{i} \in l \cap\left\{u_{d+1}=0\right\}$ for all $i$. However, $l \cap\left\{u_{d+1}=0\right\}$ is $(d-1)$-dimensional, and $\sigma^{\prime}$ is $d$-dimensional, hence this inclusion is not possible.

Assume that for some $i_{0}$ one has $a_{d+1}^{i_{0}} \neq 0$, but $a_{d+1}^{i}=0$ for all $i \neq i_{0}(1 \leqslant i \leqslant d)$. This means that $\left\{a^{i}\right\}_{i \neq i_{0}}$ is a basis for $l \cap\left\{u_{d+1}=0\right\}$. Notice that each $a_{i} \in\left(\mathbb{R}^{d+1}\right)_{\geqslant 0}^{*}$, and $0=l\left(a^{i}\right)=$ $\left\langle a^{i}, \lambda_{1}\right\rangle-a_{d+1}^{i}=\left\langle a^{i}, \lambda_{1}\right\rangle$ for $i \neq i_{0}$. This can happen only if $\# I\left(\lambda_{1}\right)=1$, i.e. $\lambda_{1,1} \neq 1$, but $\lambda_{1, i}=0$ for $i \geqslant 2$. In this case $l \cap\left\{u_{d+1}=0\right\}=\mathbb{Z}\left(e^{2}, \ldots, e^{d}\right)$. Since $\# I\left(\beta+\lambda_{1}\right) \geqslant 2$, there exists an index $i^{\prime} \geqslant 2$ such that $\left\langle e^{i^{\prime}}, \beta\right\rangle=\beta_{i^{\prime}}>0$. Therefore, there exists at least one index $i_{0}^{\prime} \neq i_{0}$ such that $\left\langle a^{i_{0}^{\prime}}, \beta\right\rangle>0$. Therefore, $\gamma_{i_{0}}>0$ and $\gamma_{i_{0}^{\prime}}>0$ for some $i_{0}^{\prime} \neq i_{0}$, hence $\# I(\gamma) \geqslant 2$.

\section{Generalities about the zeta function}

\subsection{Definitions/notations}

Let $f:\left(\mathbb{C}^{d+1}, 0\right) \rightarrow(\mathbb{C}, 0)$ be the germ of an analytic function, $F_{\epsilon}=f^{-1}(\epsilon) \cap B_{r}$ the Milnor fiber of $f$, $m_{\text {geom }}$ the geometric monodromy acting on $F_{\epsilon}$ (defined up to an isotopy), and $m_{q} \in \operatorname{Aut} H_{q}\left(F_{\epsilon}, \mathbb{R}\right.$ ) the algebraic monodromy induced by $m_{\text {geom }}(q \geqslant 0)$. Then the rational function

$$
\zeta(f)(t):=\prod_{q \geqslant 0} \operatorname{det}\left(I-t m_{q}\right)^{(-1)^{q}}
$$

is called the zeta function of $f$.

If, additionally, one has a local analytic divisor $(V, 0) \subset\left(\mathbb{C}^{d+1}, 0\right)$, then one can take a geometric monodromy $m_{\text {geom }}$ compatible with $(V, 0)$. This means that $m_{\text {geom }}$ acts on $\left(F_{\epsilon}, F_{\epsilon} \cap V, F_{\epsilon} \backslash V\right)$. This gives rise to two additional fibrations (over the circle) with fibers $F_{\epsilon} \cap V$, respectively $F_{\epsilon} \backslash V$. The corresponding zeta functions are denoted as follows:

$$
\begin{gathered}
\zeta\left(\left.f\right|_{V}\right)(t)=\prod_{q \geqslant 0} \operatorname{det}\left(I-\left.t m_{\text {geom }, q}\right|_{H_{q}\left(F_{\epsilon} \cap V, \mathbb{R}\right)}\right)^{(-1)^{q}}, \\
\zeta(\{f=\epsilon\} \backslash V)(t)=\prod_{q \geqslant 0} \operatorname{det}\left(I-\left.t m_{\text {geom }, q}\right|_{H_{q}\left(F_{\epsilon} \backslash V, \mathbb{R}\right)}\right)^{(-1)^{q}} .
\end{gathered}
$$

By a Mayer-Vietoris argument one has:

$$
\zeta(f)=\zeta\left(\left.f\right|_{V}\right) \cdot \zeta(\{f=\epsilon\} \backslash V) .
$$

In particular, if $f, g:\left(\mathbb{C}^{d+1}, 0\right) \rightarrow(\mathbb{C}, 0)$ are germs of hypersurface singularities, then taking $V=$ $\{g=0\}, \zeta\left(\left.f\right|_{g=0}\right)$ means $\zeta\left(\left.f\right|_{V}\right)$. 
Notice that the 'degree' of the rational function $\zeta(f)(t)$ is exactly the Euler characteristic $\chi\left(F_{\epsilon}\right)$ of $F_{\epsilon}$. Sometimes we denote this number by $\chi(f)$. Similarly, the degree of $\zeta\left(\left.f\right|_{g=0}\right)$ will be denoted by $\chi\left(\left.f\right|_{g=0}\right)$ (which is the Euler characteristic of the Milnor fiber $\{f=\epsilon\} \cap\{g=0\} \cap B_{r}$ of the restriction $\left.\left.f\right|_{g=0}\right)$. The following example will be a building block in the proof of Theorems $\mathrm{A}$ and $\mathrm{B}$.

Example 4.1. Let $f:\left(\mathbb{C}^{d+1}, 0\right) \rightarrow(\mathbb{C}, 0)$ be defined by $f\left(x, x_{d+1}\right)=x^{\beta}\left(x_{d+1}^{n_{1}}+c x^{\lambda}\right)$, where $x=$ $\left(x_{1}, \ldots, x_{d}\right), x^{\lambda}=x_{1}^{\lambda_{1}} \cdots x_{d}^{\lambda_{d}}$ (similarly for $\left.x^{\beta}\right), n_{1} \geqslant 1$, and $c \in \mathbb{C}$. Assume that $\# I(\beta) \geqslant 1$ and $\# I(\beta+\lambda) \geqslant 2$. (Here, as above, $I(\alpha)=\left\{i \mid \alpha_{i}>0\right\}$.) Then $\zeta(f)(t)=1$.

Indeed, since $f$ is given by a weighted homogeneous polynomial, we can replace the local situation by the affine one. Then $F_{\epsilon}=\{f=\epsilon\} \subset \mathbb{C}^{d+1}$. Assume that $c \neq 0$, and consider the projection $p r: \mathbb{C}^{d+1} \rightarrow \mathbb{C}^{d}$ given by $\left(x, x_{d+1}\right) \mapsto x$. Then for $\epsilon \neq 0, \operatorname{pr}\left(F_{\epsilon}\right)=\mathbb{C}^{d} \backslash \bigcup_{\beta_{j} \neq 0}\left\{x_{j}=0\right\}$. The discriminant locus $\Delta$ of the projection $p r: F_{\epsilon} \rightarrow \operatorname{pr}\left(F_{\epsilon}\right)$ is $\left\{c x^{\beta+\lambda}=\epsilon\right\}$. Since $I(\beta) \geqslant 1$, one gets $\chi\left(\operatorname{pr}\left(F_{\epsilon}\right)\right)=0$; similarly, since $I(\beta+\lambda) \geqslant 2, \chi(\Delta)=0$. Therefore, $\chi\left(F_{\epsilon}\right)=0$. Notice that the above geometric picture is compatible with the monodromy action, hence $\zeta(f)=1$ by similar argument (cf. [Ném91, Lemma 3.3.9]). (If $c=0$ then the result is trivial.)

4.2 One has the following generalization of A'Campo's formula [A'C75], which provides the zeta function in terms of an embedded resolution.

Fix the following data:

i) an arbitrary analytic germ $h:\left(\mathbb{C}^{d+1}, 0\right) \rightarrow(\mathbb{C}, 0)$;

ii) a local analytic divisor $(V, 0) \subset\left(\mathbb{C}^{d+1}, 0\right)$;

iii) an analytic subset $(S, 0) \subset(V, 0) \cup(\{h=0\}, 0)$.

Let $B_{r}$ be a sufficiently small ball in $\mathbb{C}^{d+1}$ centered at the origin. We write $\{h=\epsilon\}$ for the Milnor fiber $h^{-1}(\epsilon) \cap B_{r}(0<\epsilon \ll r)$.

Assume that $\phi: X \rightarrow B_{r}$ is a birational modification such that:

1) $\phi^{-1}(\{h=0\} \cup V)$ is a normal crossing divisor;

2) $\phi$ is an isomorphism above $B_{r} \backslash S$.

Let $E$ be the total transform of $\{h=0\} \cup V,\left\{E_{i}\right\}_{i}$ the irreducible components of $E$, and $m_{E_{i}}(h)$ the vanishing order of $h \circ \phi$ along $E_{i}$.

Proposition 4.2. With the above notations, one has:

$$
\zeta(\{h=\epsilon\} \backslash V)=\prod_{i}\left(1-t^{m_{E_{i}}(h)}\right)^{\chi\left(E_{i} \backslash \bigcup_{j \neq i} E_{j}\right)} .
$$

The proof is similar to the proof of the classical case [A'C75] (see also [AGV88, Theorem 3.10]), and it is left to the reader. The classical case corresponds to $V=\emptyset$ (cf. also with Remark 4.6.)

Corollary 4.3 (Splitting property for $\chi$ ) [BMcN02]. Let $f, g:\left(\mathbb{C}^{d+1}, 0\right) \rightarrow(\mathbb{C}, 0)$ be two germs of analytic functions. Then

$$
\chi(f g)=\chi(f)+\chi(g)-\chi\left(\left.f\right|_{g=0}\right)-\chi\left(\left.g\right|_{f=0}\right) .
$$

In order to emphasize the similarities and the differences between the splitting property of $\chi$ and $\zeta$, we present a short proof of Corollary 4.3.

Proof. Fix an embedded resolution $\phi$ of the divisor $(\{f g=0\}, 0) \subset\left(\mathbb{C}^{d+1}, 0\right)$ which has the property that it is an isomorphism above $\mathbb{C}^{d+1} \backslash S$ for some $S \subset \operatorname{Sing}\{f g=0\}$. Let $E$ be the total transform of $\{f g=0\}$. By applying Proposition 4.2 for $h=f$ and $V=\{g=0\}$, we obtain $\chi(f)-\chi\left(\left.f\right|_{g=0}\right)=$ $\sum_{i} m_{E_{i}}(f) \cdot \chi\left(E_{i} \backslash \bigcup_{j \neq i} E_{j}\right)$. 


\section{J. MCEWAN AND A. NÉmethi}

One can write a similar identity for $\chi(g)-\chi\left(\left.g\right|_{f=0}\right)$, and for $\chi(f g)$ (with $h=f g$ and $V=\emptyset$ ). The corollary then follows from $m_{E_{i}}(f g)=m_{E_{i}}(f)+m_{E_{i}}(g)$.

4.3 Notice that Corollary 4.3 has no analog for $\zeta$, since, in general, $\left(1-t^{m_{E_{i}}(f)}\right)^{\chi_{i}} \cdot\left(1-t^{m_{E_{i}}(g)}\right)^{\chi_{i}} \neq$ $\left(1-t^{m_{E_{i}}(f g)}\right)^{\chi_{i}}$. In fact, the reader can easily find examples of germs $f, g$ such that $\zeta(f g) \neq$ $\zeta(f) \zeta(g) / \zeta\left(\left.f\right|_{g=0}\right) \zeta\left(\left.g\right|_{f=0}\right)$.

Nevertheless, we prove the following weak version of the splitting property for $\zeta$.

Proposition 4.4 (Weak splitting property for $\zeta$ ). Let $f, g:\left(\mathbb{C}^{d+1}, 0\right) \rightarrow(\mathbb{C}, 0)$ be two germs of analytic functions. Assume that for any $k \geqslant 1$ we have $\zeta\left(f^{k} g\right)(t)=1$. Then

$$
\zeta(f)=\zeta\left(\left.f\right|_{g=0}\right) \quad \text { and } \quad \zeta(g)=\zeta\left(\left.g\right|_{f=0}\right) .
$$

Proof. Consider the geometric set-up of the proof of Corollary 4.3. By Proposition 4.2 one gets

$$
\frac{\zeta(f)}{\zeta\left(\left.f\right|_{g=0}\right)}=\prod_{i}\left(1-t^{m_{E_{i}}(f)}\right)^{\chi_{i}},
$$

where $\chi_{i}:=\chi\left(E_{i} \backslash \bigcup_{j \neq i} E_{j}\right)$; and there is a similar formula for $\zeta(g) / \zeta\left(\left.g\right|_{f=0}\right)$. Moreover,

$$
\zeta\left(f^{k} g\right)=\prod_{i}\left(1-t^{k m_{E_{i}}(f)+m_{E_{i}}(g)}\right)^{\chi_{i}} .
$$

Now the result follows from the next lemma (with $s=1$ ). If the pairs $\left\{m_{E_{i}}(f), m_{E_{i}}(g)\right\}_{i}$ are not distinct, regroup them replacing $\left\{\chi_{i}\right\}_{i}$ by $\left\{\bar{\chi}_{j}:=\sum_{i \in I_{j}} \chi_{i}\right\}_{j}$ for convenient index sets $\left\{I_{j}\right\}_{j}$ corresponding to equal pairs.

Lemma 4.5. Let $J$ be a finite set, $s \in \mathbb{Z}_{>0},\left\{\bar{\chi}_{j}\right\}_{j \in J}$ a set of integers, and finally $m_{l}^{j}>0(j \in$ $J, 0 \leqslant l \leqslant s)$ positive integers such that the vectors $m^{j}=\left(m_{0}^{j}, \ldots, m_{s}^{j}\right) \in \mathbb{Z}_{>0}^{s+1}$ are distinct $(j \in J)$. Assume that for any $\left(k_{1}, \ldots, k_{s}\right) \in \mathbb{Z}_{>0}^{s}$ we have:

$$
\prod_{j \in J}\left(1-t^{m_{0}^{j}+\sum_{l \geqslant 1} m_{l}^{j} k_{l}}\right)^{\bar{\chi}_{j}}=1 .
$$

Then $\bar{\chi}_{j}=0$ for all $j \in J$.

Proof. If $m^{j_{0}}$ is maximal in the lexicographic order, then

$$
M:=m_{0}^{j_{0}}+\sum_{l \geqslant 1} m_{l}^{j_{0}} k_{l}>m_{0}^{j}+\sum_{l \geqslant 1} m_{l}^{j} k_{l}
$$

for any $j \neq j_{0}$ and some well-chosen $k_{l}$ 's. Fix such $k_{l}$ 's, take a primitive $M$-root $\xi$ of unity, and see the multiplicity of $t-\xi$ in the left- and right-hand sides of (15). This gives $\bar{\chi}_{j_{0}}=0$. Then proceed by induction.

Remark 4.6. In Proposition 4.2 it was convenient to formulate the A'Campo type theorem in terms of the total transform. However, when $V=\emptyset$, it is more natural to formulate it in terms of the compact space $\phi^{-1}(0)$. In fact, if we stratify the spaces considered in Proposition 4.2 with respect to $\phi^{-1}(0)$ and its complement $\phi^{-1}\left(B_{r} \backslash\{0\}\right)$, the contribution to the zeta function over $B_{r} \backslash\{0\}$ is 1 by vanishing of some Euler characteristics. Therefore, it is enough to consider only the compact space $\phi^{-1}(0)$. In fact, this will be crucial in the proof of the main theorems. On the other hand, in some cases, we need a similar result formulated in the context of partial resolutions. This type of statement was proved in [GLM97].

Theorem 4.7. Let $\phi: X \rightarrow B_{r}$ be an arbitrary birational modification such that $\phi$ is an isomorphism above the complement of $\{f=0\}$. Let $\mathcal{S}$ be a semi-analytic stratification of $\phi^{-1}(0)$ such that 
along each stratum $\Xi$ of $\mathcal{S}$ the zeta function of the germ $(f \circ \phi, x)$ at $x \in \Xi$ does not depend on $x \in \Xi$. Denote this rational function by $\zeta_{\Xi}(t)$. Then:

$$
\zeta(f)(t)=\prod_{\Xi \in \mathcal{S}} \zeta_{\Xi}(t)^{\chi(\Xi)} .
$$

(In this paper stratification means a pre-stratification, without any regularity assumption.)

Remark 4.8. In the setting of an embedded resolution when the total transform is a normal crossing divisor (and the stratification is a "natural' one), then all the contributions from the non-topdimensional strata $\Xi$ vanish, since along them $\zeta_{\Xi}(t)=1$. (In fact this follows from Proposition 4.2, case $c=0$. Compare also with Proposition 4.2 or with the original case of $\mathrm{A}^{\prime} \mathrm{Campo}\left[\mathrm{A}^{\prime} \mathrm{C} 75\right]$.)

\section{The proofs of Theorems A and B}

\subsection{Proof of Theorem B}

TheOREM B (reformulated). Let $f$ be a quasi-ordinary singularity as in $\S 2.1$. We fix $\beta \in \mathbb{Z}_{\geqslant 0}^{d}$ with $\# I(\beta) \geqslant 1$. Assume that $g>0$ and the first distinguished tuple $\lambda_{1}$ satisfies $\# I\left(\beta+\lambda_{1}\right) \geqslant 2$. Then

$$
\zeta\left(x^{\beta} f\right)=1 \text {. }
$$

Proof. First notice that we can assume that $f$ satisfies property (4) of $\S 2.2((1)$ and (2) are automatically satisfied, cf. $\S 2.2$ ). Then consider a regular fan $\Sigma$ as in Proposition 3.2, and the partial resolution $\pi(\Sigma): Z(\Sigma) \rightarrow \mathbb{C}^{d+1}$ as described in $\S 3$. The critical locus of $\pi(\Sigma)$ is included in both $F$ and $F_{1}$ (cf. Proposition 3.2). Therefore, Theorem 4.7 can be applied for both $x^{\beta} f$ and $x^{\alpha} f_{1}$ (for some $\alpha$, see below). First we apply for the germ $x^{\beta} f$.

Define the index set

$$
\Sigma^{(1)}(\beta):=\Sigma^{(1)} \backslash\left\{e^{i}\right\}_{i \notin I(\beta)}
$$

The corresponding divisor is

$$
D(\beta):=\bigcup_{a \in \Sigma^{(1)}(\beta)} D(a) .
$$

Obviously, $D(\beta) \subset D$, and $D(\beta) \cup \tilde{F}$ is exactly the total transform of $x^{\beta} f$.

The divisor $D$ has two natural stratifications provided by intersections of type $\left\{D_{A}\right\}$, where in the first case $A \subset \Sigma^{(1)}$, and in the second case $A \subset \Sigma^{(1)}(\beta)$. Obviously, the second one is coarser.

In fact, in this proof we will need the following 'mixed stratification' of $D$ :

$$
\left\{\left(\bigcap_{a \in A} D(a) \backslash \bigcup_{a \notin A} D(a)\right) \backslash \tilde{F}\right\}_{A \subset \Sigma^{(1)}(\beta)},\left\{\left(\bigcap_{a \in A} D(a) \backslash \bigcup_{a \notin A} D(a)\right) \cap \tilde{F}\right\}_{A \subset \Sigma^{(1)}} .
$$

(The first group of strata has the advantage that it is compatible with A'Campo's theorem in the sense that $\zeta_{\Xi}(t)=1$ for all non-top-dimensional strata $\Xi$; cf. Remark 4.8; the second group has the advantage that it corresponds to a toroidal embedding, hence $\chi(\Xi)=0$ for all non-zerodimensional strata $\Xi$; cf. (14). See the details below.)

This stratification of $D$ induces a stratification on $\pi(\Sigma)^{-1}(0)$ which will be denoted by $\mathcal{S}$. By Lemma 3.3, each stratum $\Xi$ of $\mathcal{S}$ is included in some compact intersection of type $\bigcap_{a \in A} D(a)$. By the very definition, the stratification is compatible with $\tilde{F}$. Denote by $\mathcal{S} \cap \tilde{F}$ (respectively by $\mathcal{S} \backslash \tilde{F}$ ) the collection of those strata which are in $\tilde{F}$ (respectively are in $D \backslash \tilde{F}$ ). The stratification is compatible with the assumptions of Theorem 4.7 by $\S 3.8$. Then

$$
\zeta\left(x^{\beta} f\right)(t)=\prod_{\Xi \in \mathcal{S} \backslash \tilde{F}}\left(\zeta_{\Xi}(t)\right)^{\chi(\Xi)} \cdot \prod_{\Xi \in \mathcal{S} \cap \tilde{F}}\left(\zeta_{\Xi}(t)\right)^{\chi(\Xi)} .
$$




\section{J. MCEWAN AND A. NÉmethi}

The first factor on the right-hand side of (16), which we denote $\zeta_{\mathcal{S} \backslash \tilde{F}}$, corresponds to the product over $\Xi \in \mathcal{S} \backslash \tilde{F}$. It can be computed as follows. Since $D(\beta)$ is the total transform of $x^{\beta} f$, and the divisor $D(\beta) \backslash \tilde{F}$ is a normal crossing divisor, one gets that for any $\Xi \in \mathcal{S} \backslash \tilde{F}$ the corresponding contribution is

$$
\zeta_{\Xi}(t)^{\chi(\Xi)}=\left(1-t^{m \Xi\left(x^{\beta} f\right)}\right)^{\chi(\Xi)},
$$

provided that $\operatorname{dim} \Xi=d$, and equals 1 otherwise (cf. Remark 4.8). If $\operatorname{dim} \Xi=d$, then $\Xi \subset D(a)$ for some $a \in \Sigma^{(1)}$, and by (11) we obtain

$$
m_{\Xi}\left(x^{\beta} f\right)=m_{D(a)}\left(x^{\beta} f\right)=m(a)+\langle a, \beta\rangle=\inf _{v \in \mathcal{N}(f)}\langle a, v\rangle+\langle a, \beta\rangle .
$$

Therefore

$$
\zeta_{\mathcal{S} \backslash \tilde{F}}=\prod\left(1-t^{m(a)+\sum_{l} a_{l} \beta_{l}}\right)^{\chi(\Xi)},
$$

where the product is over $\Xi \in \mathcal{S} \backslash \tilde{F}$ with $\Xi \subset D(a)$ (and $1 \leqslant l \leqslant d$ ).

Next, for any $k=\left(k_{1}, \ldots, k_{d}\right) \in \mathbb{Z}_{>0}^{d}$, consider the germ

$$
x^{k \cdot \beta} f_{1}:=x_{1}^{k_{1} \beta_{1}} \cdots x_{d}^{k_{d} \beta_{d}} \cdot f_{1} .
$$

We apply Theorem 4.7 for this germ and modification $\pi(\Sigma)$.

The main point is that the same stratification $\mathcal{S}$ is good in this case as well (cf. Proposition 3.5). Moreover, $D \cup\left\{\right.$ total transform of $\left.x^{k \cdot \beta} f_{1}\right\}$ is a normal crossing divisor (cf. $\S 3.6$ ), hence in the $\zeta\left(x^{k \cdot \beta} f_{1}\right)$ we have contributions only from the top-dimensional strata, and all of these are situated in $\mathcal{S} \backslash \tilde{F}$.

On the other hand, we know from Example 4.1 that $\zeta\left(x^{k \cdot \beta} f_{1}\right)=1$. Therefore:

$$
\prod\left(1-t^{\left(1 / e_{1}\right) m(a)+\sum_{l} a_{l} k_{l} \beta_{l}}\right)^{\chi(\Xi)}=1
$$

for any $\left(k_{1}, \ldots, k_{d}\right) \in \mathbb{Z}_{>0}^{d}$, and the product is over $\Xi \in \mathcal{S} \backslash \tilde{F}$ with $\Xi \subset D(a)$ (and $1 \leqslant l \leqslant d$ ). In this formula we used for a stratum $\Xi \subset D(a)$ that

$$
m_{\Xi}\left(f_{1}\right)=\inf _{v \in \mathcal{N}\left(f_{1}\right)}\langle a, v\rangle=\inf _{v \in\left(1 / e_{1}\right) \mathcal{N}(f)}\langle a, v\rangle=\frac{1}{e_{1}} m_{\Xi}(f) .
$$

But (17), (18) and Lemma 4.5 imply that in (16) the first factor $\zeta_{\mathcal{S} \backslash \tilde{F}}=1$.

Now, the second factor on the right-hand side of (16) is a product over $\Xi \in \mathcal{S} \cap \tilde{F}$. By Proposition 3.5 and (14), each positive dimensional stratum has Euler characteristic $\chi(\Xi)=0$. Hence, (16) reads as

$$
\begin{aligned}
\zeta\left(x^{\beta} f\right)(t) & =\prod_{\operatorname{dim} \Xi=0, \Xi \in \mathcal{S} \cap \tilde{F}} \zeta_{\Xi}(t) \\
& =\prod_{\sigma^{\prime} \subset \Sigma \cap l, \operatorname{dim} \sigma^{\prime}=d} \zeta\left(\text { total transform of } x^{\beta} f \text { at } O_{\sigma^{\prime}}\right)(t) .
\end{aligned}
$$

But each total transform at $O_{\sigma^{\prime}}$ has the form $u^{\gamma} \tilde{f}_{\sigma}$ satisfying all the inductive properties: $\tilde{f}_{\sigma}$ is quasiordinary in local coordinates $\left(u, w_{1}\right)$ with properties (1), (2) and (4), it has less complexity (i.e. with $g-1$ distinguished tuples), and $\# I(\gamma) \geqslant 1, \# I\left(\gamma+\lambda_{1}\left(\tilde{f}_{\sigma}\right)\right) \geqslant 2$ (cf. $\S 3.10$ and Proposition 3.7).

Notice that, in the case $g=1, u^{\gamma} \tilde{f}_{\sigma}$ has the form $u^{\gamma} w$ in some local coordinates $(u, w)$, hence by Example 4.1 its zeta function is 1 . Hence Theorem B follows by induction (over $g$ ).

\subsection{Proof of Theorem A}

Theorem $\mathrm{A}$ is a consequence of Theorem $\mathrm{B}$ and the splitting property. Indeed, we can assume that $f$ satisfies properties $(1)-(4)$ (cf. $\S 2.2)$. Set $\beta:=(0, \ldots, 0, k)$. Then $\# I(\beta) \geqslant 1$ and $\# I\left(\beta+\lambda_{1}\right) \geqslant 2$. Then by Theorem B one has $\zeta\left(x_{d}^{k} f\right)=1$ for any $k \geqslant 1$. Then $\zeta(f)=\zeta\left(\left.f\right|_{x_{d}=0}\right)$ by Proposition 4.4. 


\subsection{Final remarks}

1) In the case of an irreducible plane curve singularity (i.e. in the case of $d=1), \zeta(f)$ is a complete topological invariant. If $d \geqslant 2$, then this is not true any more: $\zeta(f)$ forgets almost all the information about the (normalized) distinguished tuples $\left\{\lambda_{i}\right\}_{i}$ of $f$. For example, if $\lambda_{1,2} \neq 0$, then $\zeta(f)(t)=1-t^{n}$, hence from $\zeta(f)(t)$ only the degree $n$ can be recovered. On the other hand, this fact can have interesting consequences (e.g. in the Jung program).

2) It is not true that the Milnor fiber $F_{\epsilon}(f)$ of $f$ has the same homotopy type as the Milnor fiber of $F_{\epsilon}\left(\left.f\right|_{x_{d}=0}\right)$ of $\left.f\right|_{x_{d}=0}$. For example, if $d=2$ and $f=x_{3}^{n}-x_{1} x_{2}$, then $F_{\epsilon}(f)$ is $\bigvee_{n-1} S^{2}$, but $F_{\epsilon}\left(\left.f\right|_{x_{2}=0}\right) \approx$ disjoint union of $n$ discs. Notice that $F_{\epsilon}(f)$ can be obtained from $F_{\epsilon}\left(\left.f\right|_{x_{2}=0}\right)$ by identifying the boundaries of the discs (i.e. by an $S^{1}$-equivariant surgery).

\section{REFERENCES}

Abh55 S. S. Abhyankar, On the ramification of algebraic functions, Amer. J. Math. 77 (1955), 575-592.

A'C75 N. A'Campo, La fonction zêta d'une monodromie, Comment. Math. Helv. 50 (1975), 233-248.

AGV88 V. I. Arnol'd, S. M. Gusein-Zade and A. N. Varchenko, Singularities of differentiable maps, vols I and II (Birkhäuser, Basel 1988).

BMc00 C. Ban and L. J. McEwan, Canonical resolution of a quasi-ordinary surface singularity, Can. J. Math. 52 (2000), 1149-1163.

BMcN02 C. Ban, L. J. McEwan and A. Némethi, On the Milnor fiber of a quasi-ordinary surface singularity, Can. J. Math. 54 (2002), 55-70.

EN85 D. Eisenbud and W. Neumann, Three-dimensional link theory and invariants of plane curve singularities, Ann. Math. Studies, vol. 110 (Princeton University Press, 1985).

Ful93 W. Fulton, Introduction to toric varieties, Ann. Math. Studies, vol. 131 (Princeton University Press, 1993).

Gau88 Y.-N. Gau, Embedded topological classification of quasi-ordinary singularities, Mem. Amer. Math. Soc. 388 (1988), 109-127.

GLM97 S. M. Gusein-Zade, I. Luengo and A. Melle-Hernández, Partial resolutions and the zeta-function of a singularity, Comment. Math. Helv. 72 (1997), 244-256.

GP00 P. D. González Pérez, Quasi-ordinary singularities via toric geometry, Thesis, University of La Laguna, 2000.

GT00 R. Goldin and B. Teissier, Resolving singularities of plane analytic branches with one toric morphism, in Resolution of singularities, A research textbook in tribute to Oscar Zariski, eds H. Hauser, J. Lipman, F. Oort and A. Quiros, Progress in Math. No 181 (Birkhäuser-Verlag, Basel, 2000), $315-340$.

LDT73 Lê Dũng Tráng, Topologie des singularités des hypersurfaces complexes, Astérisque 7-8 (1973), $171-182$.

Lip65 J. Lipman, Quasi-ordinary singularities of embedded surfaces, Thesis, Harvard University (1965).

Lip83 J. Lipman, Quasi-ordinary singularities of surfaces in $\mathbb{C}^{3}$, Proc. Symp. Pure Math. 40 (1983), $161-172$.

Lip88 J. Lipman, Topological invariants of quasi-ordinary singularities, Mem. Amer. Math. Soc. 388 (1988), 1-107.

Mil68 J. Milnor, Singular points of complex hypersurfaces, Ann. Math. Studies, vol. 61 (Princeton University Press, 1968).

MO70 J. Milnor and P. Orlik, Isolated singularities defined by weighted homogeneous polynomials, Topology 9 (1970), 385-393.

Ném91 A. Némethi, The Milnor fiber and the zeta function of the singularities of type $f=P(h, g)$, Compositio Math. 79 (1991), 63-97.

Ném93 A. Némethi, The zeta function of singularities, J. Algebraic Geom. 2 (1993), 1-23. 
Oda88 T. Oda, Convex bodies and algebraic geometry, Ergebnisse der Mathematic und ihner Grenzgebiete, vol. 15 (Springer-Verlag, Berlin, 1988).

Oh93 K. Oh, Topological types of quasi-ordinary singularities, Proc. Amer. Math. Soc. 117 (1993), 53-59.

Sch90 R. Schrauwen, Topological series of isolated plane curve singularities, Enseign. Math. 36 (1990), $115-141$.

Sie90 D. Siersma, The monodromy of a series of hypersurface singularities, Comment. Math. Helv. 65 (1990), 181-197.

Tei74 B. Teissier, Déformations à type topologique constant I, II; in Séminaire Douady-Verdier 1971-72, Astérisque 16 (1974), 215-249.

Var76 A. N. Varchenko, Zeta function of monodromy and Newton diagram, Invent. Math. 37 (1976), 253-262.

Yau89 S.-T. S. Yau, Topological types of isolated hypersurface singularities, Contemp. Math. 101 (1989), 303-321.

Zar68 O. Zariski, Studies in equisingularity, III. Saturation of local rings and equisingularity, Amer. J. Math. 90 (1968), 961-1023; reprinted in Collected papers, vol. 4, 96-158.

Lee J. McEwan mcewan@math.ohio-state.edu

Department of Mathematics, Ohio State University, 231 West 18th Avenue, Columbus, OH 43210, USA

András Némethi nemethi@math.ohio-state.edu Department of Mathematics, Ohio State University, 231 West 18th Avenue, Columbus, OH 43210, USA 